\title{
Prevalencia del trastorno bipolar y factores asociados en las parroquias rurales del cantón Cuenca
}

\author{
Prevalence of bipolar disorder and associated factors in the rural parishes of \\ Cuenca city
}

Carla Alexandra Idrovo Idrovo. ${ }^{1}$, Christian Miguel Moncayo Rivera. ${ }^{2}$, Wilson Daniel Lasso León. $^{3}$ \& Pastora María Rivera Hernández. ${ }^{4}$

\begin{abstract}
DOI: https://doi.org/10.33262/cienciadigital.v4i1.1105

Introduction: Bipolar disorder is a pathology of mood that is characterized by episodes that change between mania and depression; The objective of the study was to know the prevalence and associated factors of bipolar disorder in the population of the rural parishes of the Cuenca city. Materials and methods: A cross sectional analysis study was carried out. The universe was made up of 199,506 inhabitants, through a multi-stage sample 984 individuals from the parishes El Valle, Tarqui and Checa were evaluated. A sociodemographic interview and the MINI test (International Mini Neuropsychiatric Interview for adults) were applied. People 18 years of age and older were included and individuals with severe cognitive and behavioral disorders were excluded. Results: It was found that 32 people had current bipolar disorder corresponding to a prevalence of $3.30 \%$ of which $1.42 \%$, were reported as a single disorder. People suffering from mania currently accounted for $1.70 \%$ of the population; while that suffering hypomania were $1.50 \%$. A higher percentage was found in the female sex. The only associated factor is not living with a couple, with a chi-square of 11.60 (p: 0.00) with an OR of 2.20 (CI 1.39 - 3.50). There is no association with family structure and functionality, employment status, migration, age and sex. Comorbidities were considered: anxiety and dependence-abuse of alcohol and
\end{abstract}

\footnotetext{
${ }^{1}$ Ministerio de Salud Pública, carla.idrovo@ hotmail.com

${ }^{2}$ Universidad Católica de Cuenca, christianmoncayo487@gmail.com

${ }^{3}$ Hospital Homero Castanier Crespo, danielleon@ gmail.com

${ }^{4}$ Universidad Católica de Cuenca, maria_rivera123@hotmail.com
} 
substances. Conclusions: Bipolar disorder has a prevalence of 3.30\%. The main associated factor was living without a partner.

Keywords: Bipolar disorder, anxiety, comorbidity.

\section{Resumen}

Introducción: El trastorno bipolar es una patología del estado de ánimo que se caracteriza por episodios que cambian entre manía y depresión; el objetivo del estudio fue conocer la prevalencia y factores asociados del trastorno bipolar en la población de las parroquias rurales del cantón Cuenca. Materiales y métodos: Se realizó un estudio de tipo transversal analítico. El universo estuvo conformado por 199.506 habitantes, a través de una muestra polietápica se evaluaron a 984 individuos de las parroquias El Valle, Tarqui y Checa. Se aplicó una entrevista sociodemográfica y el test MINI (Mini Entrevista Neuropsiquiátrica Internacional para adultos). Se incluyó personas de 18 años en adelante y se excluyó a individuos con alteraciones cognitivas y conductuales graves. Resultados: Se encontró que 32 personas presentaron trastorno bipolar actual que corresponde a una prevalencia del $3,30 \%$ de los cuales el 1,42\%, se reportaron como trastorno único. Las personas que sufren manía actualmente representaron el 1,70\% de la población; mientras que los que sufren hipomanía fueron el 1,50\%. Se encontró un mayor porcentaje en el sexo femenino. El único factor asociado es el no convivir con una pareja, con un chi cuadrado de 11,60 (p: 0.00) con un OR de 2,20 (IC 1,39-3,50). No existe asociación con estructura y funcionalidad familiar, situación laboral, migración, edad y sexo. Se consideró como comorbilidades: ansiedad y dependencia-abuso de alcohol y sustancias. Conclusiones: El trastorno bipolar tiene una prevalencia del 3,30\%. El principal factor asociado fue vivir sin una pareja.

Palabras clave: Trastorno bipolar, ansiedad, comorbilidad.

\section{Introducción}

El trastorno bipolar es una afección psiquiátrica, crónica, distinguida por un mal control de las emociones ocasionando cuadros de depresión mayor, manía e hipomanía en el individuo; es muy peligrosa porque no es igual a las disparidades que sienten todas las personas, más bien causa 
disminución de la funcionalidad social, laboral e interpersonal y los síntomas son tan potentes que pueden conducir al suicidio. (1) (2) (3)

El trastorno bipolar a su vez tiene 2 subtipos: bipolar I y bipolar II; el paciente con bipolaridad tipo I manifiesta síntomas maniacos y la mayoría de veces presentan depresión mayor e hipomanía; a diferencia del bipolar tipo II que no manifiesta episodios de manía, pero si depresión e hipomanía. (3)

Generalmente la depresión mayor suele ser el principal problema al inicio de la enfermedad con un $54 \%$, seguido de la manía con un $22 \%$ y un patrón mixto (depresión y manía) un 24\%. (4) Sus principales síntomas son: la disforia, bajo interés por actividades placenteras, poca energía, existe alteración de la concentración y falta de memoria, un lenguaje lento y suave, pérdida o aumento de peso, es común también una afectación del sueño produciéndose insomnio o hipersomnia, la persona tiende a sentirse inútil o culpable con ideas o conductas suicidas en algunos casos, cuida poco su apariencia personal e higiene, tiene ideología pesimista, desesperanza e indecisión. (4) (5) (6)

El diagnóstico de desorden bipolar tipo I se realiza en personas con uno o más episodios de manía y al menos un episodio de depresión mayor e hipomanía, mientras que el de bipolar tipo II se realiza en personas con antecedentes de al menos un episodio de hipomanía y de depresión mayor, pero sin haber tenido periodos de manía. Los principales diagnósticos diferenciales son: esquizofrenia, trastorno de hiperactividad con déficit de atención, trastorno esquizoafectivo y trastorno de personalidad. (7)

La psicoterapia y farmacoterapia de mantenimiento es pilar fundamental del tratamiento. Los fármacos usados comúnmente son: litio, anticonvulsivos, antipsicóticos como monoterapia o combinados, para potenciar el efecto y reducir las crisis. (8)

Los benzodiacepinas se usan principalmente como tratamiento adyuvante para el insomnio, agitación, o ansiedad. (9)

Una investigación realizada en todo el mundo basado en la Encuesta de Salud Mental de la OMS indica tasas de prevalencia de trastorno bipolar a lo largo de la vida de alrededor de un $2.4 \%$ específicamente un $0.6 \%$ para bipolaridad tipo I, un $0,4 \%$ para a bipolaridad tipo II y un $1.4 \%$ para formas subclínicas del trastorno bipolar. Mientras que Goodwin indica que este desorden 
afecta al $1.2 \%$ de los habitantes de la población del mundo y su edad de inicio preferente es la juventud (2)

Sin embargo, las tasas de prevalencia por año para el trastorno bipolar son del $1.5 \%$, un $0.4 \%$ para el tipo I, un $0.3 \%$ para el tipo II y un $0,8 \%$ para las otras formas de bipolaridad. Estas cifras dependen de las variaciones en los criterios diagnósticos en cuanto a tiempo y del área el 2\%, pero al considerar los otros estados de bipolaridad ascendió al 6\%. (3)

La proporción de hombres y mujeres que desarrollan el trastorno bipolar es de aproximadamente 1: 1. Un análisis de salud mental de Colombia refleja que el trastorno bipolar tipo I se presenta en el 1,8\% de la población y el 0,2 \% tiene bipolaridad tipo II. La prevalencia a lo largo de la vida en Medellín es del 1,4\% según el Estudio de Salud Mental realizado entre 2011 y 2012.(4)

En cuanto a las edades de inicio para las dos enfermedades son similares.; el trastorno bipolar I (manía) con 20 años y trastorno bipolar II (hipomanía) con 24 años. (10)

Una encuesta del año 1994 respecto a los trastornos psiquiátricos en la población urbana de México mostró una prevalencia del Trastorno bipolar de 0.65\%. (11)

Según un informe de la OPS sobre salud mental del año 2004 señala que en nuestro país de todos los pacientes que han ingresado en los cinco hospitales psiquiátricos existentes un $17 \%$ corresponden a trastornos del ánimo; mientras que de las personas que acuden a consulta externa un $25,7 \%$ padece bipolaridad siendo predominante en varones menores de 17 años. (12)

En la ciudad de Cuenca una investigación realizada en el CRA en adultos de 18 a 65 años indica una prevalencia del $1.85 \%$ de TB tipo I y un 0,02\% del TB tipo II; siendo la manía la presentación clínica predominante. (13)

El objetivo del estudio es identificar la prevalencia y factores asociados del trastorno bipolar en la población de las parroquias rurales del cantón Cuenca, lo cual nos ayudará a tener una orientación sobre la situación de salud mental en la ciudad ya que no existen datos concretos.

\section{Materiales y métodos}

El enfoque elegido correspondió a un estudio de tipo transversal analítico. El universo de esta investigación estuvo conformado por 199.506 habitantes. La muestra fue polietápica, aleatorizada, conformada por 984 individuos de las parroquias El Valle, Tarqui y Checa, las cuales fueron seleccionadas considerando su representatividad de acuerdo a la ubicación geográfica y 
crecimiento poblacional. A dicha población se aplicó una encuesta sociodemográfica y el test MINI (Mini Entrevista Neuropsiquiátrica Internacional para adultos). Los datos obtenidos fueron procesados y analizados en el programa SPSS. Se identificó a quienes presentan o presentaron trastorno bipolar; se estableció la distribución del trastorno de acuerdo a las variables: estructura y funcionalidad familiar, convivencia de pareja, situación laboral, migración, edad, sexo y su asociación.

Se estratificó de acuerdo a la edad, para lo cual se consideraron cuatro estratos 18-20, 21-40, 4165 y de 65 y más; a continuación, en cada grupo de edad se estratificó de acuerdo al sexo considerando para ello la distribución establecida por el INEC en el censo 2010.

Para la ubicación de los individuos en el área geográfica se aleatorizaron todas las viviendas de las parroquias de acuerdo a los estratos edad y sexo. En caso de que la vivienda aleatorizada no contenga la unidad de análisis otorgada, se utilizó el criterio del vecino más cercano.

En el presente estudio se incluyeron a todas las personas que se encuentren entre los 18 años en adelante, que manifiesten voluntad de participar en la entrevista y firmar consentimiento informado. Se excluyeron a individuos que tenga dificultad para comprender la encuesta, y que presenten alteraciones cognitivas y conductuales graves.

\section{Resultados}

Se realizó un estudio transversal con una muestra polietápica de 984 individuos residentes en las parroquias El Valle, Checa y Tarqui del Cantón Cuenca de los cuales, 47\% (438) correspondieron al sexo masculino y el 53\% (546) al femenino. La media de la edad en los hombres fue de 43.93 (DS 19.56) años; la mediana fue 40.5 para un valor mínimo de 18 y máximo de 90 . En las mujeres la media fue de 41.72 (DS 17.30); la mediana estuvo en 39 con una edad mínima de 18 y máxima de 93. Se obtuvo la prevalencia de punto de los trastornos actuales obteniéndose el 20.73\% (IC 18.31 - 23.38). El 13.62\% (IC 11.62 - 15.90) presentó más de un trastorno (prevalencia de comorbilidades). 
Tabla 1. Prevalencia actual de trastornos mentales (únicos) en la población rural del cantón Cuenca, Ecuador. 2018

\begin{tabular}{lcc}
\hline \multicolumn{1}{c}{ TRASTORNO } & No & \% \\
\hline EDM & 95 & 9,65 \\
Distimia & 6 & 0,61 \\
Bipolar & 14 & 1,42 \\
Angustia actual & 11 & 1,12 \\
Agorafobia & 9 & 0,91 \\
Fobia social & 11 & 1,12 \\
Obsesivo compulsivo & 5 & 0,51 \\
Estrés postraumático & 12 & 1,22 \\
Dependencia al alcohol & 13 & 1,32 \\
Abuso de alcohol & 9 & 0,91 \\
Dependencia de sustancias & 0 & 0 \\
Abuso de sustancias & 0 & 0 \\
Psicótico actual & 10 & 1,02 \\
Ansiedad generalizada & 9 & 0,91 \\
Total & $\mathbf{2 0 4}$ & $\mathbf{2 0 , 7 3}$ \\
\hline
\end{tabular}

Fuente: Datos Estadísticos SPSS

Elaborado por: Los investigadores

Del total de personas, que fueron estudiadas en el intervalo de tiempo de enero del 2018 a junio del mismo año, 32 presentaron trastorno bipolar actual que implica una prevalencia del 3,30\% de los cuales, 14 (1,42\%), se reportaron con este trastorno como único. Desagregando, lo maníacos actuales representaron el 1,70\% (17) de la población; los hipomaníacos actuales fueron 15 (1,50\%). La prevalencia de trastorno bipolar pasado afectó al 5,0\% (49) de los individuos: 29 $(2,95 \%)$ no refirieron otro trastorno adicional a lo largo de la vida. Quienes padecieron episodio maníaco fueron 24 (2,40\%) individuos y el episodio hipomaníaco afectó a 25 (2,50\%) individuos.

Tabla 2. Trastorno bipolar en habitantes de la zona rural del cantón Cuenca, según parroquia estudiada y sexo. Cuenca, 2018

\begin{tabular}{clrrrr}
\hline \multirow{2}{*}{ Sector } & Sexo & \multicolumn{2}{c}{ Bipolar Actual } & \multicolumn{2}{c}{ Bipolar pasado } \\
& & No. & \multicolumn{1}{c}{$\%$} & \multicolumn{1}{c}{ No. } & \multicolumn{1}{c}{$\%$} \\
\hline \multirow{2}{*}{ El Valle } & Mujer & 16 & 4,9 & 12 & 3,7 \\
& Hombre & 5 & 2 & 19 & 7,6 \\
Checa & Mujer & 0 & 0 & 4 & 9,5 \\
& Hombre & 0 & 0 & 2 & 4,7 \\
Tarqui & Mujer & 5 & 2,8 & 7 & 3,9 \\
& Hombre & 6 & 4,1 & 5 & 3,4 \\
TOTAL & Mujer & 21 & 3,8 & 23 & 4,2 \\
& Hombre & 11 & 2,5 & 26 & 5,9 \\
& Total & 32 & 3,3 & 49 & 5 \\
\hline
\end{tabular}

Fuente: Datos Estadísticos SPSS

Elaborado por: Los investigadores 
Dos casos refirieron haber presentado el trastorno en el pasado y padecerlo en la actualidad. La media de la edad en las mujeres fue 35.23 (IC 30.69-39-78; SW 0.922, p 0.006) años; en los hombres fue 38.25 (IC 32.54-43.96; SW 0.911, p 0.007).

De acuerdo a las variables investigadas, la distribución de la siguiente manera:

Tabla 3. Trastorno bipolar en habitantes de la zona rural del cantón Cuenca, según variables estudiadas. Cuenca, 2018

\begin{tabular}{llcr}
\hline \multirow{2}{*}{ VARIABLES } & \multicolumn{2}{c}{ TRASTORNO BIPOLAR } \\
& No. & $\%$ \\
\hline \multirow{2}{*}{ Sexo } & Mujeres & 43 & 7,9 \\
& Hombres & 36 & 8,2 \\
Convivencia de pareja & No convive & 42 & 12 \\
& Si convive & 37 & 5,8 \\
Situación laboral & Sin remuneración & 44 & 9,7 \\
\multirow{4}{*}{ Migración familiar } & Con remuneración & 35 & 6,6 \\
Migración en el núcleo & Si & 50 & 9,4 \\
primario & No & 29 & 6,4 \\
& No & 43 & 9,4 \\
Tipo de familia & No tradicional & 36 & 6,8 \\
\multirow{4}{*}{ Funcionalidad familiar } & Tradicional & 13 & 10,3 \\
& Vive solo & 56 & 7,4 \\
& Disfuncional & 10 & 10,3 \\
& Funcional & 65 & 9,3 \\
Edad por ciclo de vida & De 18 a 20 & 15 & 7,7 \\
& De 21 a 40 & 33 & 15,5 \\
& De 41 a 65 & 28 & 8 \\
& De 65 y más & 3 & 8,9 \\
& & & 1,9 \\
\hline
\end{tabular}

Fuente: Datos Estadísticos SPSS

Elaborado por: Los investigadores

En base la distribución que se presenta en la tabla No. 3, se procedió a explorar la asociación y la significancia de la misma.

Tabla 4. Trastorno bipolar y variables relacionadas como factores de riesgo, en habitantes de la zona rural del cantón Cuenca, según variables estudiadas. Cuenca, 2018

\begin{tabular}{lrrrrr}
\hline \multicolumn{1}{c}{ Variables } & \multicolumn{1}{c}{$\begin{array}{c}\text { Chi } \\
\text { cuadrado }\end{array}$} & $\mathrm{p}$ & OR & \multicolumn{2}{c}{ IC } \\
\hline Sexo mujer & 0,39 & 0,8 & 1 & 0,6 & 1,5 \\
No convive con pareja & 11,6 & 0 & 2,2 & 1,4 & 3,5 \\
Situación laboral sin remuneración & 3,3 & 0,1 & 1,5 & 1 & 2,4 \\
Migración familiar & 2,88 & 0,1 & 1,5 & 0,9 & 2,4 \\
Migración en el núcleo primario & 2,2 & 0,1 & 1,4 & 0,9 & 2,3 \\
Tipo de familia no tradicional & 1,32 & 0,3 & 1,5 & 0,8 & 2,7 \\
Familia disfuncional & 0,15 & 0,7 & 1,2 & 0,4 & 3,6 \\
Edad en hombres 50 años y más & 1,36 & 0,2 & 0,6 & 0,3 & 1,4 \\
Edad en mujeres 43 años y más & 4,5 & 0 & 0,5 & 0,2 & 1 \\
\hline
\end{tabular}

Fuente: Datos Estadísticos SPSS

Elaborado por: Los investigadores 
El principal factor asociado con el trastorno bipolar es el no convivir con una pareja, ya que se evidencia un chi cuadrado de 11,60 (p: 0.00) con un OR de 2,20 (IC 1,39-3,50).

En torno a las comorbilidades, la distribución se presenta en la tabla siguiente.

Tabla 5. Comorbilidad del trastorno bipolar con otras condiciones mentales en habitantes de la zona rural del cantón Cuenca, 2018

\begin{tabular}{lcc}
\hline \multicolumn{1}{c}{ Comorbilidad } & No. & $\%$ \\
\hline Riesgo de suicidio leve & 14 & 17,90 \\
Riesgo de suicidio moderado & 4 & 33,30 \\
Riesgo de suicidio alto & 6 & 30,00 \\
Trastorno de ansiedad & 36 & 19,50 \\
Dependencia y abuso de alcohol y sustancias & 10 & 16,40 \\
Trastorno psicótico & 15 & 26,18 \\
\hline
\end{tabular}

Fuente: Datos Estadísticos SPSS

Elaborado por: Los investigadores

De las comorbilidades presentadas, dos se consideraron como factores de riesgo para trastorno bipolar: ansiedad y dependencia-abuso de alcohol y sustancias. En el primer caso el valor de chi cuadrado fue de 40,318 (p 0.00) con un OR de 4,248 (IC 2,64 - 6,84); con el segundo factor, el chi cuadrado fue de 6,163 (p 0,01), el OR fue 2,43 (IC 1,18-4,99).

\section{Discusión}

Los disturbios mentales son un fundamento significativo de discapacidad y morbilidad e incrementan el riesgo de sufrir otras complicaciones de salud.(14)

Dentro de las 6 causas principales de discapacidad se encuentran los trastornos psiquiátricos (depresión, trastornos por abuso de alcohol, esquizofrenia y trastorno bipolar). (15)

Además, éstos amplían el riesgo de padecer otras enfermedades como las patologías cardiovasculares o diabetes y la infección por VIH. (16)

La prevalencia en América según la OPS de dichos trastornos en general varía entre 18,7 y 24,2\%; la de los trastornos por ansiedad, entre 9,3 y $16,1 \%$; la de trastornos afectivos, entre 7,0 y 8,7\%; y la de trastornos debidos al consumo de sustancias psicoactivas, entre 3,6\% y 5,3\%. (14) 
La depresión es una de las patologías más frecuentes en todo el mundo, y se deduce que aqueja a más de 300 millones de personas.(17)

Mundialmente, se estima que 4,4\% de la población sufre depresión y 3,6\%, un trastorno de ansiedad. Esta tasa varía ligeramente entre territorios de la OMS: con respecto a la depresión, las tasas fluctúan de 3,6\% en la Región del Pacífico Occidental a 5,4\% en la Región de África, y en el caso de los trastornos de ansiedad, las tasas se estiman de 2,9\% en la Región del Pacífico Occidental a 5,8\% en la Región de las Américas. (18)

En nuestro estudio encontramos una prevalencia total de trastornos mentales del 20,73\% que se encuentra dentro de los parámetros hallados a nivel de América que nos indica la OPS, además dentro de las enfermedades más frecuentes encontramos que la depresión alcanza una cifra de $9,65 \%$ que duplica el porcentaje hallado a nivel mundial, la ansiedad generalizada en un $0,91 \%$ con una cifra menor para lo estimado mundialmente y el continente americano; sin embargo al contrario de lo esperado no se encontró un valor significativo para el trastorno de abuso de sustancias.

El trastorno bipolar es una enfermedad crónica que se caracteriza por eventos que varían entre la manía y la depresión. Según Goodwin citado por Echeverri (2), esta enfermedad afecta al 1,2\% de la población mundial; mientras que la OMS, citado por Echeverri (2), sostiene que la afección se da en un 2,4\%. No obstante, al llevar esta realidad al grupo de personas estudiadas, que son los habitantes de las parroquias rurales de Cuenca, se observó que la prevalencia se da en el 3,30\% de los pacientes; casi tres veces el porcentaje establecido por Goodwin (2); y prácticamente un 50\% más de lo indicado por la OMS.

La OMS (2) sostiene que la bipolaridad tipo I se presenta en mayor cantidad de casos que la bipolaridad tipo II. Se recuerda que la principal diferencia entre ambas es que la bipolaridad tipo I presenta episodios maniacos severos, mientras que la bipolaridad tipo II presenta eventos hipomaniacos; los cuales no son tan graves como los primeros. Esto coincide con los resultados encontrados en la población rural de Cuenca, donde la cantidad de personas con bipolaridad tipo I es mayor a la de bipolaridad tipo II.

En lo referente a la distribución de casos de bipolaridad por edades, se muestra que la enfermedad suele presentarse en la adultez; alrededor de los 21 años. En un estudio reciente efectuado en 11 países se halló que la mediana de la edad de comienzo es a los 25 años. (19) 
Particularmente en Colombia, se establece que la edad más usual en la cual se presenta por primera vez la enfermedad es 24 años. Sin embargo, esto no es dato estático debido a que anterior a esta edad es complicado diagnosticar la enfermedad, ya que los adolescentes suelen presentar episodios hipomaniacos como consecuencia de causas diferentes a la bipolaridad (4).

En la investigación realizada se constató que, efectivamente, la edad más prevalente para el trastorno es en la adultez joven. Para las personas de 21-40 años, se presentaron 33 casos de bipolaridad; mientras que para los adolescentes menores a 21 años se presentaron 15 casos. Se debe recordar que el trastorno tarda hasta 8 años en hacerse presente, por lo que puede que los adolescentes presenten episodios leves de hipomanía, pero aún no se clasifican con bipolaridad. En lo relacionado a las personas de la tercera edad, sólo se registraron 3 casos que sufrían de bipolaridad. Sin embargo, esto puede estar relacionado a lo establecido en el estudio del Ministerio de Salud de Colombia (6), donde se establece que los síntomas de la bipolaridad pueden ser tan fuertes que podrían llegar a conducir al suicidio. Una suposición válida por hacer es que hay pocas personas de tercera edad con bipolaridad porque algún porcentaje se suicidad debido a los estragos de la enfermedad antes de cumplir los 65 años.

En relación a la distribución según el sexo, la literatura presentada por Infobae. (20) indica que el trastorno bipolar tipo I se presenta de igual manera en hombres como en mujeres; mientras que el trastorno bipolar tipo II se presenta en casi el doble de casos en mujeres que en hombres. Esto se podría decir que va de la mano con lo investigado en el estudio presentado debido a que se encontró un mayor número de mujeres afectadas con bipolaridad. Si bien es cierto que no se hizo distinción entre bipolaridad I o II, hay un mayor número de mujeres que padecen de la afección en general. Esto es algo nuevo para la medicina. Según una publicación realizada por Infobae (20), no fue hasta hace pocos años que las mujeres se empezaron a ver más afectadas que los hombres por la bipolaridad, llegando a ser esta relación de 2:1 (20)

El motivo de esto no es especificado claramente en ninguna referencia literaria confiable que se pueda encontrar, pero sin duda es algo que se ve reflejado en las parroquias rurales de Cuenca y empieza a observarse en el mundo.

En lo relacionado al trabajo, debido a que las personas que padecen bipolaridad tienen dificultad para relacionarse con otros es común que no puedan mantener trabajos estables. De hecho, esto no 
sólo se ve reflejado en la vida laboral, sino que en ocasiones las personas bipolares no pueden mantener un lugar en las universidades o colegios. (21)

Esto no es diferente en la población de parroquias rurales de Cuenca. Los voluntarios en el estudio muestran que la mayor parte de los que padecen bipolaridad no tienen un trabajo que les genere una remuneración consistente.

Lidiar con una pareja bipolar es algo que requiere de mucha preparación y comprensión. La bipolaridad suele generar problemas en la sexualidad de los que la padecen, además crea impulsividad al momento de tomar decisiones al momento de gastar el dinero. Estos dos son temas de usual controversia en las parejas que tienen a una persona que padece un trastorno mental como la bipolaridad; ya que son mucho más difíciles de manejar (21). No se encontró ningún estudio en particular que otorgue cifras concretas sobre la vida de pareja de las personas bipolares, sin embargo, debido a las complicaciones que podría haber no es extraño suponer que pueden producirse rupturas de pareja debido a las dificultades de la bipolaridad.

En el estudio realizado en las parroquias rurales de Cuenca se encontró que la mayor parte de las personas que padecen bipolaridad no tienen una pareja con la cual convivir. No obstante, prácticamente todos viven en una familia tradicional y tienen un núcleo familiar funcional, algo opuesto ya que se espera que dichos individuos arrastren una disfuncionalidad en su hogar. Esto puede significar que las personas bipolares mantienen mayormente una relación funcional con sus padres o hermanos, a pesar de no conseguir mayormente una pareja estable.

En una investigación realizada en el 2017, se encontró que el 61,9 \% de los pacientes con trastorno bipolar tenían adicionalmente un trastorno por uso de sustancias y se supusieron duales, con mayor comorbilidad con trastornos de ansiedad y un mayor riesgo de suicidio, que se ha relacionado con la posibilidad de factores genéticos comunes. (22) (23)

En nuestro estudio se observó algo similar ya que las dos principales comorbilidades encontradas fueron el trastorno de abuso de sustancias y el trastorno de ansiedad, por lo que no se debería descartar algún patrón genético.

Los datos obtenidos servirán como base para posteriores estudios y el porcentaje arrojado como prevalencia es de utilidad para establecer la probabilidad de que un paciente que llega a la consulta tenga desorden bipolar sin haber aplicado algún test o examen complementario. 


\section{Conclusiones}

El trastorno bipolar instituye un desorden mental, con un gran impacto a nivel social y familiar que se presenta con mayor particularidad en el sexo femenino, la prevalencia en las parroquias rurales del cantón Cuenca es el 3,30\%.

El principal factor asociado fue vivir sin una pareja. No se encontró asociación con la estructura y funcionalidad familiar, situación laboral, migración, edad y sexo. Se consideró como comorbilidades: ansiedad y dependencia-abuso de alcohol y sustancias.

\section{Referencias Bibliográficas}

(1) Miller T. Bipolar disorder. Elsevier. 2016 Jun; 43(2): 269-84

(2) Echeverri P. "Investigación sobre el trastorno afectivo bipolar". Revista Cúpula [Online]. [cited 2017 mayo. Available from: http://www.binasss.sa.cr/bibliotecas/bhp/cupula/v22n1/art3.pdf.

(3) García A, Sierra P, Lorenzo L. Nosología, epidemiología y etiopatogenia del trastorno bipolar: Últimas aproximaciones. Elsevier. 2014 junio ; 21(3).

(4) Suppes,T. El trastorno bipolar en adultos: Características clínicas. UptoDate [Online].; 2016 [cited 2017 febrero. Available from: https://www.uptodate.com/contents/bipolar-disorder-inadults-clinicalfeatures?source=search $\_$result\&search=trastorno\%20bipolar\&selectedTitle $=2 \sim 150$.

(5) Lyness, J. Depresion en adultos: Evaluación y diagnóstico.UptoDate [Online].; 2016 [cited 2017 febrero. Available from: https://www.uptodate.com/contents/unipolar-depression-inadults-assessment-and-diagnosis?source=see_link.

(6) American Psychiatric Association. DSM V. In Diagnostic and statistical manual of mental disorders : DSM-5..: Arlington; 2013.

(7) Suppes T. Bipolar disorder in adults: Assessment and diagnosis. UptoDate [Online].; 2016 [cited 2017 mayo. Available from: https://www.uptodate.com/contents/bipolar-disorder-inadults-assessment-and-

$\underline{\text { diagnosis?source }=\text { search } \_ \text {result } \& \text { search }=\text { trastorno\%20bipolar } \& \text { selectedTitle }=5 \sim 150}$.

(8) Post R. Bipolar disorder in adults: Choosing maintenance treatment. UptoDate[Online].; 2017 [cited 2017 mayo. Available from: https://www.uptodate.com/contents/bipolar-disorder-inadults-choosing-maintenancetreatment?source=search _result $\&$ search $=$ trastorno\%20bipolar \&selectedTitle $=4 \sim 150$. 
(9) Stoval J. El trastorno bipolar en adultos: Tratamiento farmacológico para la manía aguda y hipomanía. UptoDate [Online].; 2016 [cited 2017 mayo. Available from: https://www.uptodate.com/contents/bipolar-disorder-in-adults-pharmacotherapy-for-acutemania-andhypomania?source=search_result\&search=tratamiento $\% 20 \mathrm{de} \% 20$ trastorno $\% 20$ bipolar\&select $\underline{\text { edTitle }=3 \sim 150}$.

(10) Ministerio de Protección Social de Colombia. Estudio Nacional de Salud Mental de Colombia. [Online].; 2003 [cited 2017 mayo. Available from: https://www.minsalud.gov.co/Documentos\%20y\%20Publicaciones/ESTUDIO\%20NACION AL\%20DE\%20SALUD\%20MENTAL\%20EN\%20COLOMBIA.pdf.

(11) Medina-Mora M. Trastornos Emocionales de la población urbana mexicana: resultados de un estudio nacional. Anales del Instituto Mexicano de Psiquiatría [Online].; 1992 [cited 2017. Available from: http://www.uade.inpsiquiatria.edu.mx/Articulos\%20Jorge/1992/Trastornos\%20emocionales\% 20en\%20poblacion\%20urbana\%20mexicana\%20resultados $\% 20$ de $\% 20$ un $\% 20$ estudio $\% 20$ naci onal.\%201992.pdf.

(12) Organizacion Panamericana de Salud. Informe sobre el sistema de salud mental en ecuador: Organizacion Panamericana de Salud. [Online].; 2009 [cited 2017 mayo. Available from: http://www.who.int/mental_health/ecuador_who_aims_report.pdf.

(13) Vintimilla J, Paredes J. Prevalencia de Trastorno Bipolar en Centro de Adicciones de Cuenca. [Online].Cuenca:Universidad del Azuay; 2011 [cited 2017 mayo. Available from: http://dspace.uazuay.edu.ec/bitstream/datos/53/1/08494.pdf.

(14) Organización Panamericana de Salud. La salud mental en la Región de las Américas: Organización Panamericana de Salud. [Online].; 2013 [cited 2019 septiembre. Available from: https://www.paho.org/salud-en-las-americas-2017/?post_t_es=la-salud-mental-en-la-regionde-las-americas\&lang=es.

(15) Organización Mundial de la Salud. Invertir en salud mental: Organización Mundial de la Salud. [Online].; 2004 [cited 2019 septiembre. Available from: https://www.who.int/mental_health/advocacy/en/spanish_final.pdf.

(16) Organización Mundial de la Salud. Datos y cifras de salud mental: Organización Mundial de la Salud. [Online].; 2017 [cited 2019 septiembre. Available from: https://www.who.int/features/factfiles/mental_health/mental_health_facts/es/index4.html. 
(17) Organización mundial de la Salud. Depresión. [Online].; 2018 [cited 2019 septiembre. Available from: https://www.who.int/es/news-room/fact-sheets/detail/depression.

(18) Organización Panamericana de la Salud. Depresión y otros trastornos mentales comunes. [Online].; 2017 [cited 2019 septiembre. Available from: http://iris.paho.org/xmlui/bitstream/handle/123456789/34006/PAHONMH17005-spa.pdf.

(19) Martinez Hernandez , Montalván Martinez O, Betancourt Izquierdo. Trastorno Bipolar. Consideraciones clínicas y epidemiológicas: Rev Médica Electrónica. [Online].; 2019 [cited 2019 septiembre.

http://www.revmedicaelectronica.sld.cu/index.php/rme/article/view/3157/4255.

(20) Infobae. Trastorno Bipolar: por qué las mujeres son las más afectadas: Infobae. [Online].; 2015 [cited 2019 Agosto 29. Available from: https://www.infobae.com/2015/03/27/1718516trastorno-bipolar-que-las-mujeres-son-las-mas-afectadas/.

(21) National Institute of Mental Health. Trastorno Bipolar. National Institute of Mental Health, Salud y Servicios Humanos; 2009. Report No.: 15-3679.

(22) Secadez- Alvarez A, Fernandez Rodriguez C. Revisión de la eficacia de los tratamientos para el trastorno bipolar en comorbilidad con el abuso de sustancias: Rev Psiquiatría y Salud Mental. [Online].; 2017 [cited 2019 septiembre. Available from: https://www.elsevier.es/es-revistarevista-psiquiatria-salud-mental286-articulo-revision-eficacia-los-tratamientos-el$\underline{\text { S1888989115001640. }}$.

(23) Carmiol N, Peralta J, Almasy. Shared genetic factors influence risk for bipolar disorder and alcohol use disorders. Eur Psychiatry. 2014; 29: p. 282- 87.

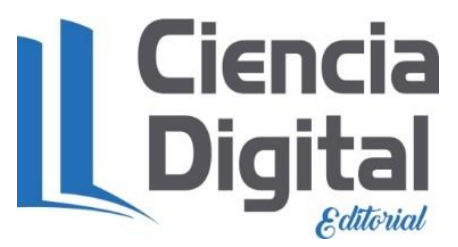




\section{PARA CITAR EL ARTÍCULO INDEXADO}

Idrovo Idrovo, C. A., Moncayo Rivera, C. M., Lasso León, W. D., \& Rivera Hernández, P. M. (2020). Prevalencia del trastorno bipolar y factores asociados en las parroquias rurales del cantón Cuenca. Ciencia Digital, 4(1), 349-363. https://doi.org/10.33262/cienciadigital.v4i1.1105

\section{Ciencia
Digital
$\varepsilon_{\text {tudtaal }}$}

El artículo que se publica es de exclusiva responsabilidad de los autores y no necesariamente reflejan el pensamiento de la Revista Ciencia Digital.

El artículo queda en propiedad de la revista y, por tanto, su publicación parcial y/o total en otro medio tiene que ser autorizado por el director de la Revista Ciencia Digital.
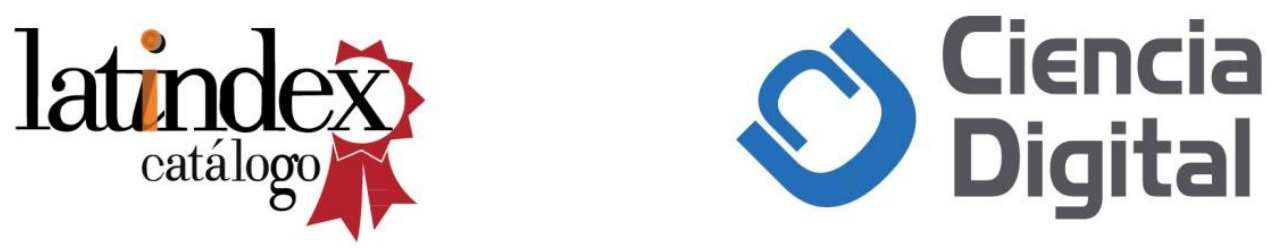